\title{
Monoallelic and biallelic CREB3L1 variant causes mild and severe osteogenesis imperfecta, respectively
}

\author{
Rachel B. Keller, BS ${ }^{1}$, Thao T. Tran, BS ${ }^{1}$, Shawna M. Pyott, PhD ${ }^{1}$, Melanie G. Pepin, MS, CGC ${ }^{1}$, \\ Ravi Savarirayan, MD, FRACP ${ }^{2,3}$, George McGillivray, $\mathrm{MBChB}^{2}$, UW Center for Mendelian Genomics, \\ Deborah A. Nickerson, $\mathrm{PhD}^{4}$, Michael J. Bamshad, $\mathrm{MD}^{4}$ and Peter H. Byers, MD ${ }^{1,5}$
}

Purpose: Osteogenesis imperfecta (OI) is a heritable skeletal dysplasia. Dominant pathogenic variants in COL1A1 and COL1A2 explain the majority of OI cases. At least 15 additional genes have been identified, but those still do not account for all OI phenotypes that present. We sought the genetic cause of mild and lethal OI phenotypes in an unsolved family.

Methods: We performed exome sequencing on seven members of the family, both affected and unaffected.

Results: We identified a variant in cyclic AMP responsive element binding protein 3-like 1 (CREB3L1) in a consanguineous family. The variant caused a prenatal/perinatal lethal OI in homozygotes, similar to that seen in OI type II as a result of mutations in type I collagen genes, and a mild phenotype (fractures, blue sclerae) in multiple heterozygous family members. CREB3L1 encodes old astrocyte specifically induced substance (OASIS), an endoplasmic reticulum stress transducer. The variant disrupts a DNA-binding site and prevents OASIS from acting on its transcriptional targets including SEC24D, which encodes a component of the coat protein II complex.

Conclusion: This report confirms that CREB3L1 is an OI-related gene and suggests the pathogenic mechanism of CREB3L1-associated OI involves the altered regulation of proteins involved in cellular secretion.

Genet Med advance online publication 17 August 2017

Key Words: bone; COPII; CREB3L1; OASIS; osteogenesis imperfecta

\section{INTRODUCTION}

Osteogenesis imperfecta (OI; MIM 166200, 166210, 259420, and 166220), is a genetically and phenotypically heterogeneous disorder characterized by brittle bones and variable bone deformity. ${ }^{1}$ Severity ranges from intrauterine fractures and significantly shortened long bones with perinatal lethality to few fractures with normal stature and life expectancy. ${ }^{1}$ Extraskeletal features may include blue sclerae, dentinogenesis imperfecta, adult-onset hearing loss, joint hypermobility, and hyperlaxity of skin. ${ }^{1}$ In the majority (>90\%) of affected individuals, mutations in COL1A1 (MIM 120150) and COL1A2 (MIM 120160), which encode the prool(I) and proo2(I) chains of mature type I procollagen, respectively, account for the phenotype. ${ }^{2}$ Genes involved in collagen processing (CRTAP (MIM 605497), P3H1 (MIM 610339), PPIB (MIM 123841), FKBP10 (MIM 607063), SERPINH1 (MIM 600943), BMP1 (MIM 112264), and PLOD2 (MIM 601865)), bone cell differentiation and signaling (SP7 (MIM 606633) and WNT1 (MIM 164820)), bone cell association with extracellular matrix (SPARC (MIM 182120)), and matrix mineralization (SERPINF1 (MIM 172860) and TMEM38B (MIM 611236)) have been implicated in rarer forms of recessively inherited disease. ${ }^{2,3}$ Additionally, mutations in IFITM5 (MIM 614757), through mechanisms that are not fully understood, cause autosomal dominant forms of OI with unique features, e.g., hyperplastic callus formation (MIM 610967). ${ }^{4,5}$ Recently, an X-linked form of OI caused by mutations in MBTPS2 (MIM 300294) was reported. ${ }^{6}$

Even with this expanded catalog of OI disease genes, the underlying cause of OI in a subset of families has still not been identified. From exome sequence analysis of members of a consanguineous family of Lebanese descent (Figure 1a), we identified a trinucleotide deletion in cyclic AMP responsive element binding protein 3-like 1 (CREB3L1; MIM 616215) that corresponds to an in-frame single amino acid deletion in old astrocyte specifically induced substance (OASIS), and results in a mild OI phenotype in heterozygotes and a lethal phenotype in homozygotes. Previously, homozygosity for whole-gene deletion of CREB3L1 was shown to result in lethal OI (MIM 616229) in a Turkish family. ${ }^{7}$ Our study confirms that mutations in CREB3L1 result in an OI phenotype wherein disease severity is determined by whether mutations are monoallelic or biallelic, and identifies a potential mechanism of action.

\section{MATERIALS AND METHODS}

\section{Exome and confirmatory sequencing}

Written informed consent was obtained for all individuals in the study. Exome sequencing was performed by the

${ }^{1}$ Department of Pathology, University of Washington, Seattle, Washington, USA; ${ }^{2}$ Victorian Clinical Genetics Services, Murdoch Children's Research Institute, Melbourne, Victoria, Australia; ${ }^{3}$ Department of Paediatrics, University of Melbourne, Melbourne, Victoria, Australia; ${ }^{4}$ Center for Mendelian Genomics, University of Washington, Seattle, Washington, USA; ${ }^{5}$ Department of Medicine (Medical Genetics), University of Washington, Seattle, Washington, USA. Correspondence: Rachel B. Keller (keller88@u.washington.edu) 
a



C

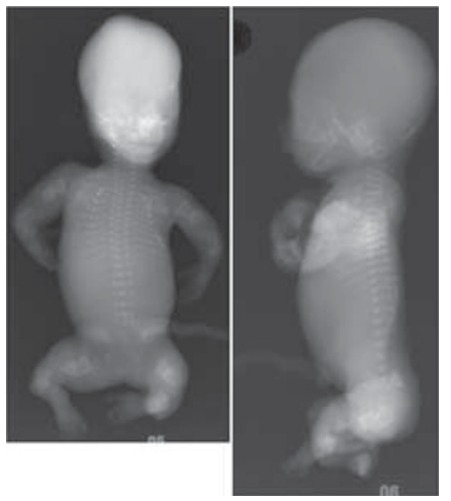

d

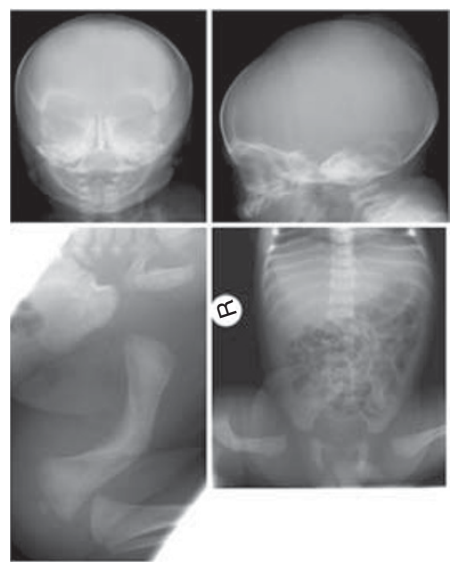

b
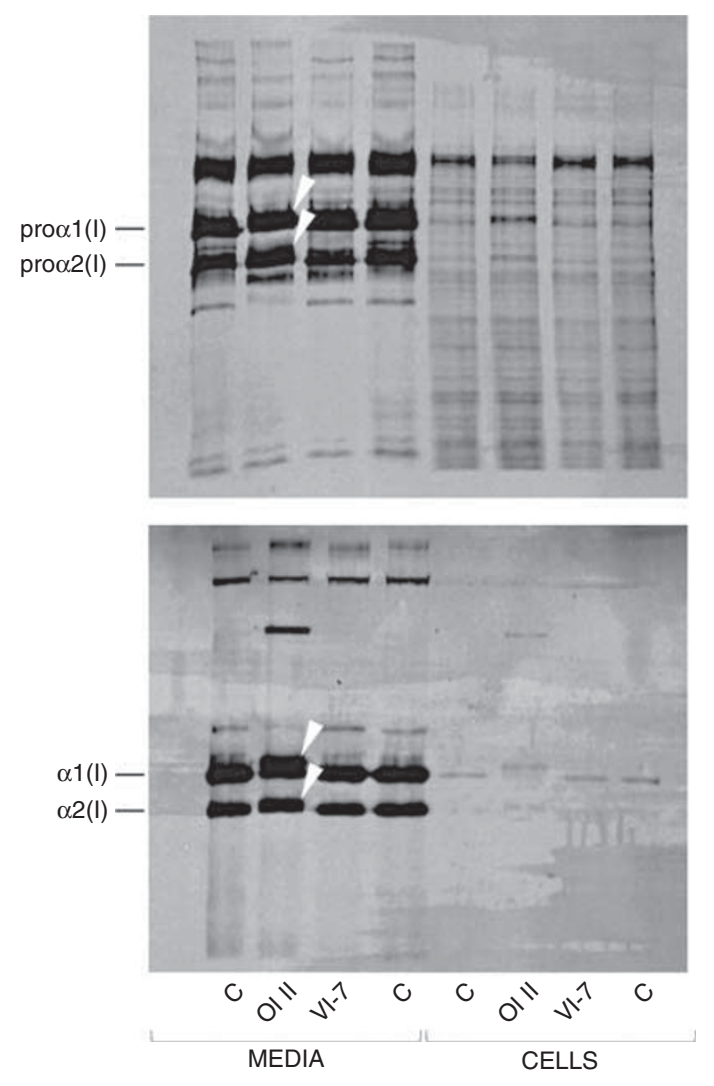

Figure 1 Homozygosity and heterozygosity for a 3-bp deletion in CREB3L1 that results in a single amino acid deletion in OASIS (c.934_936delAAG [p.Lys312del]) are consistent with the variable severity of osteogenesis imperfecta (OI) phenotypes. (a) Pedigree of the CREB3L1 Ol family. Arrow indicates the proband. Asterisks denote the family members whose exomes were sequenced. Chromatograms from Sanger sequence analysis confirming the exome findings are shown. (b) Effect of the CREB3L1 variant on type I collagen biochemistry. Collagen alpha chains synthesized by skin fibroblasts from the proband (VI-7) are structurally equivalent to those from control fibroblasts (c), in contrast to those from a typical OI type II patient (OI II) with a COL1A1 glycine substitution (p.Gly818Cys), in which overmodification results in both delayed procollagen mobility (upper panel; arrows) and delayed $\alpha 1(I)$ and $\alpha 2(I)$ chain mobility when procollagens are digested with pepsin (lower panel; arrows). We do not see abnormal retention of procollagens nor of alpha chains in the proband fibroblasts; as skin fibroblasts do not express CREB3L1, we would not expect OASIS to have a role in regulating secretion in these particular cells. (c) Anterior-posterior (left) and lateral (right) radiographs from VI-3 (homozygous for the 3bp deletion in CREB3L1) following 18 weeks gestation termination. There is virtually no calvarial mineralization. Bones in all visible extremities are telescoped and very irregular. The ribs are thin and wavy and there is mild platyspondyly. (d) Radiographs of VI-4 (heterozygous for the 3-bp deletion in CREB3L1) at birth. The calvarium is thin and the anterior and posterior fontanelles are large, but there are no Wormian bones. The ribs are thin and there is mild platyspondyly. There is marked bowing of the right femur.

University of Washington Center for Mendelian Genomics as previously described. ${ }^{8}$ Variants were sorted as described in the text. The variant in CREB3L1 was confirmed with Sanger sequencing. Primers for amplification of exon 7 are listed in Supplementary Table S1 online (Integrated DNA Technologies, Coralville, IA). Exon 7 was amplified using AmpliTaq Gold Polymerase (Applied Biosystems, Foster City, CA). The program used for cycling was: $95^{\circ} \mathrm{C}$ for 12 minutes, $95^{\circ} \mathrm{C}$ for 10 seconds, $61^{\circ} \mathrm{C}$ for 40 seconds, $72{ }^{\circ} \mathrm{C}$ for 50 seconds for 35 cycles, then, $72{ }^{\circ} \mathrm{C}$ for 7 minutes. Amplicons were treated with ExoSAP according to a standard protocol. Sequencing reactions were set up using Big Dye v3.1 (Applied Biosystems) with the following program: $96^{\circ} \mathrm{C}$ for 10 seconds, $50^{\circ} \mathrm{C}$ for 5 seconds, $60^{\circ} \mathrm{C}$ for 4 minutes for 40 cycles. Sequencing was run on an ABI 3730. Sequences were analyzed using Mutation Surveyor v4.0.9 software (Softgenetics, State College, PA).

\section{Collagen analysis}

Culture of skin fibroblasts and analysis of procollagens was performed as previously described. ${ }^{9}$ 


\section{In vitro transcription/translation and western blot}

In vitro transcription/translation (IVT) was performed using the 1-Step Human Coupled IVT Kit-DNA (ThermoFisher Scientific, Waltham, MA). Templates for IVT were generated using a polymerase chain reaction (PCR)-based method. ${ }^{10}$ Wild-type (WT) and variant (VT312) full-length CREB3L1 complementary DNA sequences were amplified from plasmids "WT312" and "MU312," generated for overexpression (OE) experiments in mammalian cell lines (see "Overexpression" below). Primers for creation of IVT templates are listed in Supplementary Table S1 (Integrated DNA Technologies, Coralville, IA). IVT reactions were assembled according

to kit instructions and incubated for 6 hours at $30^{\circ} \mathrm{C} .1 \mu \mathrm{l}$ of each IVT reaction was run on $10 \%$ sodium dodecyl sulfatepolyacrylamide gel. IVT with no DNA input or with a construct bearing green fluorescent protein DNA (pCFE-GFP; ThermoFisher Scientific) was included as (-) and (+) controls, respectively, for IVT. Detection by western blot was performed with the following primary antibodies: OASIS (sc-514635; Santa Cruz Biotechnology, Santa Cruz, CA) at 1:1000 and TurboGFP (PA5-22688; ThermoFisher Scientific) at 1:10 000. Secondary antibodies used were goat anti-mouse secondary IgG (TA130004; OriGene, Rockville, MD) and goat anti-rabbit IgG H\&L (ab6721; Abcam, Cambridge, MA), both at 1:20 000. Detection was achieved using Amersham ECL Western Blotting Detection Reagent (GE Healthcare, Pittsburgh, PA).

\section{Electrophoretic mobility shift assay}

Electrophoretic mobility shift assay (EMSA) was performed using the Gel Shift Assay Core System (Promega, Madison, WI). WT and VT312 IVT OASIS proteins were incubated with a 40 -bp fragment of the COL1A1 promoter that was synthesized as a duplex oligonucleotide (Integrated DNA Technologies, Coralville, IA). Oligonucleotide sequences are listed in Supplementary Table S1. The oligonucleotides were labeled with $\gamma^{32} \mathrm{P}$ ATP (PerkinElmer, Waltham, MA) using T4 polynucleotide kinase. Unincorporated label was removed using Micro Bio Spin P-30 Gel Columns (BIORAD, Redmond, WA). Protein and labeled oligonucleotide (and in the case of competition samples, unlabeled oligonucleotide) were incubated together at $4^{\circ} \mathrm{C}$ for 1 hour. Samples were run on a $6 \%$ nondenaturing polyacrylamide gel. Gel was dried for 4 hours at $72{ }^{\circ} \mathrm{C}$ and exposed to film overnight.

\section{Overexpression}

A human complementary DNA open reading frame clone with CREB3L1 placed under a cytomegalovirus promoter (RC204968) was purchased from OriGene (Rockville, MD) along with an empty pCMV6-Entry vector (PS100001) as a transfection control. The CREB3L1 expression construct is referred to herein as "WT312." The family deletion (VT312) was introduced using the QuickChange II Site-Directed Mutagenesis Kit to create "MU312." Primers for site-directed mutagenesis were purchased as PAGE-purified primers and are listed in Supplementary Table S1 (Integrated DNA Technologies, Coralville, IA). Constructs were transiently transfected into HEK293 using XtremeGene 9 Transfection Reagent (Roche, Indianapolis, IN) at a 3:1 ( $\mu$ l reagent: $\mu \mathrm{g}$ plasmid) ratio and incubated for 48 hours before harvest. For the dimerization experiment, "WT312" and "MU312" were transfected at the indicated ratios equaling $1 \mu \mathrm{g}$ total of plasmid $(3: 1=0.75 \mu \mathrm{g}$ WT312 $+0.25 \mu \mathrm{g}$ MU312; $1: 1=0.5 \mu \mathrm{g}$ $\mathrm{WT} 312+0.5 \mu \mathrm{g} \quad \mathrm{MU} 312, \quad 1: 3=0.25 \mu \mathrm{g} \quad \mathrm{WT} 312+0.75 \mu \mathrm{g}$ MU312).

\section{Quantitative reverse transcription PCR}

For quantitative reverse transcription PCR (RT-qPCR), total RNA was isolated from cells using the RNeasy Mini Kit (Qiagen, Valencia, CA). First-strand complementary DNA synthesis of $1 \mu \mathrm{g}$ of DNAseI-treated RNA was performed using iScript Reverse Transcription Supermix (BIORAD, Hercules, CA) in 20- $\mu$ l reactions. One $\mu$ l of complementary DNA was used for RT-qPCR per $20-\mu$ l reaction using iTaq Universal SYBRGreen Supermix (BIORAD). Samples were run on a CFX96 Touch (BIORAD) using the following program: $95^{\circ} \mathrm{C}$ for 30 seconds, $95^{\circ} \mathrm{C}$ for 5 seconds, $60{ }^{\circ} \mathrm{C}$ for 30 seconds for 40 cycles. Each sample was run in triplicate and the mean $\mathrm{Ct}$ values were analyzed using the $2^{\wedge}$-[delta] [delta]Ct method. ${ }^{11}$ Expression was normalized to the housekeeping gene GAPDH. Primers for RT-qPCR are listed in Supplementary Table S1(Integrated DNA Technologies, Coralville, IA).

\section{Immunoblotting}

Protein lysates were prepared from transfected cells using a buffer containing $0.05 \mathrm{M}$ Tris- $\mathrm{HCl}$ at $\mathrm{pH} 8.0,0.15 \mathrm{M} \mathrm{NaCl}$, $5.0 \mathrm{mM}$ EDTA, $1 \% \mathrm{NP}-40$, and a protease inhibitor cocktail at $4{ }^{\circ} \mathrm{C}$. $50 \mu \mathrm{g}$ of lysate was loaded per lane on a $10 \%$ sodium dodecyl sulfate-polyacrylamide gel in loading buffer containing $7.7 \mathrm{mg} / \mathrm{ml}$ dithiothreitol. Detection for western blot was performed with primary antibodies to SEC24D (ab191566; Abcam, Cambridge, MA) at 1:2,000, SEC23A (ab179811; Abcam) at 1:100, OASIS (ab33051; Abcam) at 1:1,000, and GAPDH (ab37168; Abcam) at 1:2,000. The secondary antibodies goat anti-rabbit IgG H\&L (ab6721; Abcam) and goat anti-mouse secondary IgG (TA130004; OriGene, Rockville, MD) were used at a 1:20,000 dilution. Detection was achieved using Amersham ECL Western Blotting Detection Reagent (GE Healthcare, Pittsburgh, PA). Quantitation of blot signals was achieved using ImageJ. ${ }^{12}$ Adjusted signal intensities were obtained by normalizing to GAPDH signal to control for loading differences between lanes.

\section{Statistical analysis}

Statistical analysis was performed using GraphPad Prism 7.0a software (http://www.graphpad.com). The RT-qPCR data are expressed as the mean $+/-\mathrm{SD}$ from three independent experiments. Statistical significance was determined by running analysis of variance and the indicated tests for multiple comparisons (see figure legends). 


\section{RESULTS}

\section{Case report}

The proband (VI-7, Figure 1) was identified on ultrasound at 12 weeks gestation due to shortened and angulated humeri, multiple fractures of the long bones of both legs, and apparently reduced skull mineralization. Spine and ribs appeared normal. Imaging at 14 weeks confirmed micromelia and angulated long bones. The ribs were normal length, but appeared angulated, consistent with fractures. Hypomineralization of the skull was not convincing at this time. At 16 weeks, the pregnancy was terminated due to suspected OI. Molecular genetic analysis of DNA extracted from skin fibroblasts excluded mutations in all genes known at the time to cause OI. Biochemical analysis of cultured fibroblasts from VI-7 demonstrated normal amounts and mobility of type I collagen and procollagen in contrast to altered electrophoresis due to overmodification of type I collagen chains from a typical OI type II patient with a heterozygous COL1A1 mutation that resulted in substitution of a triple helical glycine (p.Gly818Cys) (Figure 1b).

Two prior pregnancies had been terminated because of in utero findings consistent with severe skeletal dysplasia. The first of these pregnancies (VI-3) (Figure 1c) was terminated at 18 weeks. Radiographs showed severe long bone deformity with crumpling, angulation, and marked rhizomelic and mesomelic shortening. The ribs were fractured, but not beaded in appearance. The calvarial bones were paper thin and soft. There was almost no ossification of the cranial vault and severe demineralization was noted. There were no Wormian bones identified. Detailed clinical information on the second of the previous affected pregnancies (VI-6) was not available. An earlier pregnancy (VI-2) was lost to fetal demise at 27 weeks. The cause was unclear, but fetal lungs showed pulmonary hypoplasia.

Three individuals in the family appeared to be mildly affected and exhibited some OI-like features. The proband's brother (VI-4) (Figure 1d) had a fractured right femur at birth with acute angulation in the midshaft. Otherwise, long bones, ribs, and thorax were normal in length and shape. He did not have Wormian bones. He was treated for vitamin D deficiency postnatally (levels were $22 \mathrm{nmol} / \mathrm{L}$; lower limit of the normal range was $50 \mathrm{nmol} / \mathrm{L}$ ), as was his mother. X-ray of the pelvis at 6 weeks of age showed osteopenia. At 15 years of age, he had blue sclerae and osteopenia, but no additional fractures. The affected sister (VI-5) had blue sclerae and mild osteopenia, which was diagnosed at 2 years of age on X-ray of the knee when the long bones were also noted to be gracile, but she has no history of fractures. The mother (V-4) had blue sclerae and had recurrent fractures in childhood, including a fractured pelvis with minimal trauma at age 1 and a spiral fracture of one femur at age 16. Her father, too (IV-1), had recurrent fractures. A great grandfather of the proband (III-4) and a maternal granduncle (IV-5) reportedly had fractures and may also be mildly affected (Supplementary Figure S1). The family members in generation IV were not available for clinical assessment.

\section{Variant identification}

Exome sequence analysis was performed on samples from seven family members: V-3, V-4, VI-1, VI-4, VI-5, VI-7, and VI-8. We identified 56,634 variants that differed from the consensus genomic sequence (GRCh37/hg19) among the seven individuals. We removed variants in intergenic and intronic regions, variants found in dbSNP, and variants found more than 10 times in the Exome Sequencing Project Exome Variant Server. This reduced the list to 4,254 variants. Variants shared by affected members of the family and the unaffected siblings were discarded, leaving 659 variants. We then looked for changes that were homozygous in the proband (72 variants), but that were heterozygous in the mildly affected siblings. This left six genes as candidates: CREB3L1, FADS2, UQCC3, CTSF, MMP17, and C11orf49 (Table 1). We found a 3-bp in-frame deletion (c.934 936delAAG [p.Lys312del]) in exon 7 of the CREB3L1 gene that segregated with the phenotypes in the family (Figure 1a). While the proband (VI-7) is homozygous for the deletion, all mildly affected individuals (V-4, VI-4, VI-5) are heterozygous for the change. The two healthy siblings (VI-1 and VI-8) are homozygous for the normal allele. The father (V-3), who is reportedly unaffected, is also heterozygous. Either his phenotype is so mild it has not come to clinical attention or the mutation is not penetrant in him. He declined multiple offers of clinical assessment. The lysine residue that is lost is highly conserved (genomic evolutionary rate profiling ${ }^{13}$ $=4.69$; PhastCons $\left.{ }^{14}=1\right)($ Figure 2).

Expression of CREB3L1 has been described in a select few cell types thus far, including in osteoblasts. ${ }^{15-18}$ The protein product of CREB3L1, OASIS, is synthesized as a rough endoplasmic reticulum transmembrane protein with the $\mathrm{N}$-terminal end (residues 1-374) in the cytoplasmic compartment. The N-terminal end contains a basic leucine zipper domain and an overlapping nuclear localization signal. The C-terminal end (residues 394-519) is resident in the rough endoplasmic reticulum (Figure 2). ${ }^{15}$ Upon detection of endoplasmic reticulum (ER) stress, proteolytic cleavage releases the $\mathrm{N}$-terminal fragment. This fragment is transported to the nucleus, where it functions as a transcription factor. ${ }^{15}$ Target genes of OASIS vary among cell types. ${ }^{15-18}$ Knockout of Creb3l1 in mice results in growth retardation and fractures. Microcomputed tomography of these mice revealed low bone mineral density in femoral trabecular bone. By this and additional bone metrics, Murakami et al. ${ }^{16}$ concluded that Creb3 $311^{-1-}$ mice suffer from severe osteopenia. Based on the mouse study, on the previous report of lethal OI caused by CREB3L1 deletion, ${ }^{7}$ and on the genetics of the family described here, the variant identified in CREB3L1 was thought to be the cause of both mild and severe forms of OI.

Based on the literature, the remaining five genes-FADS2 (MIM 606149), UQCC3 (MIM 616097), CTSF (MIM 603539), MMP17 (MIM 602285), and C11orf49-were no longer reasonable candidates. FADS2 encodes delta- 6 desaturase, which is involved in the synthesis of long-chain polyunsaturated fatty acids. Single-nucleotide polymorphisms in delta- 6 


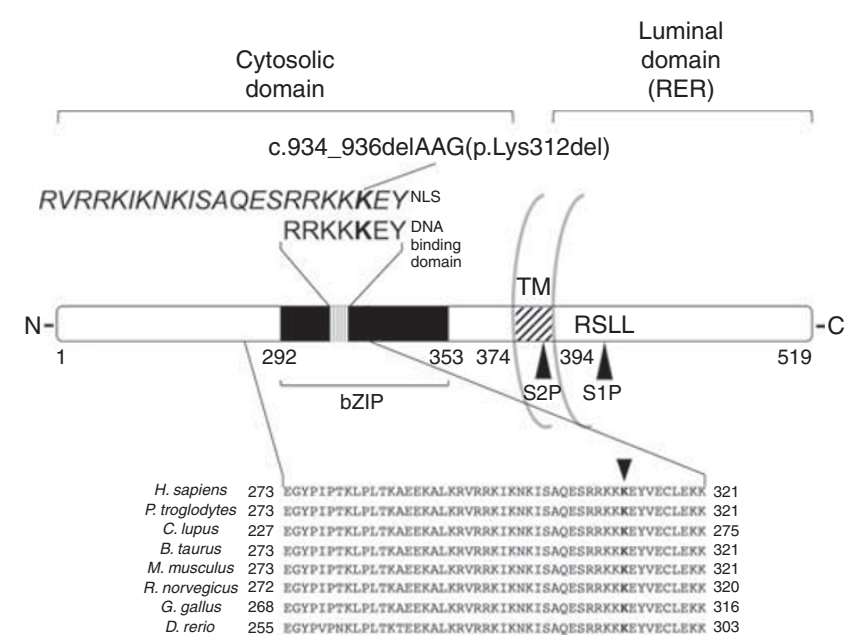

Figure 2 Old astrocyte specifically induced substance (OASIS). OASIS is a 519aa protein with a basic leucine zipper domain common to other CREB/ATF family members and a transmembrane domain that anchors it in the rough endoplasmic reticulum membrane. When endoplasmic reticulum stress challenges the cell, the full-length OASIS protein is freed from the endoplasmic reticulum membrane and is cleaved through regulated intramembrane proteolysis (RIP) at the S1P and S2P sites. The conserved DNA-binding domain is shown, with the deleted lysine residue in bold. Italics above show an overlapping predicted bipartite nuclear localization sequence. The deleted lysine residue is conserved in vertebrates (genomic evolutionary rate profiling $=4.69$; PhastCons = 1). bZIP, basic leucine zipper; NLS, nuclear localization sequence; RER, rough endoplasmic reticulum; TM, transmembrane.

desaturase and other fatty acid desaturases have been associated with a heightened risk of cardiovascular disease, including coronary artery disease and ischemic stroke. ${ }^{19}$ UQCC3 (ubiquinol-cytochrome C reductase complex assembly factor 3) is required for complex III assembly. Mutations in UQCC3 cause mitochondrial complex III deficiency, nuclear type 9. ${ }^{20}$ CTSF encodes cathepsin F, a lysosomal degradation enzyme implicated in type B Kufs disease, a neuronal ceroid lipofuscinosis. ${ }^{21}$ MMP17 encodes matrix metalloproteinase 17, an enzyme that is able to cleave extracellular matrix components. Variants in MMP17 are thought to predispose to aortic aneurysm ${ }^{22}$ and $M M P 17$ is expressed in some cancers. ${ }^{23,24}$ C11orf49 encodes a protein of unknown function.

\section{Effect of the variant on OASIS function}

Fibroblasts from the proband (VI-7) were available, but CREB3L1 is expressed at such low levels in skin ${ }^{15,16}$ we could not examine the altered function of the protein in those cells. To study the effect of the mutation, we performed IVT of WT and variant (VT312) CREB3L1 sequences. With IVT, VT312 CREB3L1 was expressed and produced a stable, full-length $(\sim 80 \mathrm{kDa})$ OASIS protein (Figure 3a). The deletion is located within both a predicted bipartite nuclear localization signal (RVRRKIKNKISAQESRRKKKEY) $)^{25}$ and a DNA-binding motif (RRKKKEY) shared among CREB/ATF family members $^{26}$ (Figure 2). Disruption of either of these domains could 
a

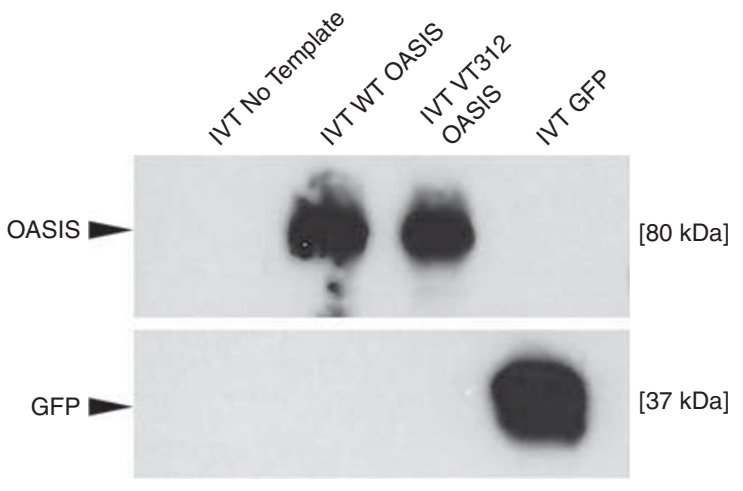

b



Figure 3 Effect of the variant on OASIS function. (a) In vitro transcription/translation (IVT). The variant protein (VT312) is stably expressed and does not appear truncated compared with wild-type (WT) protein. IVT with no DNA input or with a construct bearing green fluorescent protein DNA was included as $(-)$ and $(+)$ controls for in vitro transcription, respectively. Western blot. (b) The family variant causes a defect in DNA binding. A shift is seen when IVT WT OASIS is incubated with the radiolabeled oligo containing the unfolded protein response element-like sequence (lane 1). This shift is competed away when unlabeled oligo is added (lane 2). The shift is absent when radiolabeled oligo is incubated with IVT VT312 OASIS (lane 3). Background bands are likely binding interactions between labeled oligo and proteins from the HeLa lysate used to perform IVT. Electrophoretic mobility shift assay. GFP, green fluorescent protein; UPRE, unfolded protein response element.

prevent OASIS from transducing its targets due to either failure of the active $\mathrm{N}$-terminal fragment to reach the nucleus or to interference with DNA-binding to gene-regulatory elements, respectively.

To determine if DNA binding was affected by the deletion, we performed an EMSA to assess binding of OASIS to an unfolded protein response element-like sequence (CGACGTGG) in the COL1A1 promoter to which OASIS was previously shown to bind. ${ }^{16}$ While the WT OASIS protein bound the unfolded protein response element-like sequence, demonstrated by a band shift, no shift was detected after incubation with the Lys312del protein, indicating that the variant protein did not bind to DNA (Figure $3 \mathbf{b}$ ). On this basis, we concluded that the mutation is pathogenic.
We attempted immunocytochemistry in transfected cells treated with ER stress-inducing drugs (e.g., tunicamycin) to look at the effect of the variant on the subcellular localization of OASIS; however, we were unable to obtain reliable staining (data not shown). As the EMSA shows that the DNA-binding ability of VT312 OASIS is eliminated, we decided determination of the effect of the mutation on localization was unnecessary. Whether VT312 OASIS can reach its nuclear targets or not, it cannot bind them.

\section{OASIS is a regulator of cellular secretion}

Another CREB/ATF family member with high sequence similarity to OASIS, BBF2H7 (BBF2 human homolog on chromosome 7, encoded by CREB3L2 (MIM 608834)), was found to regulate expression of SEC23A (SEC23 homolog A; MIM 610511), a component of the inner wall of the coat protein II (COPII) complex..$^{27}$ COPII carriers are involved in the trafficking of secreted proteins from the rough endoplasmic reticulum to the Golgi. ${ }^{28}$ To determine whether OASIS also regulates SEC23A, we overexpressed WT and VT312 OASIS separately in HEK293 cells (Supplementary Figure S2) and looked for an effect on SEC23A expression. Though the data showed a slight increase in expression of SEC23A in the presence of VT312 OASIS $(P=0.03)$, there was neither significant difference in SEC23A messenger RNA levels between WT overexpression and the empty vector control, nor between WT and VT312 overexpression. We concluded that expression of SEC23A was not affected (Figure 4a), consistent with the previously published finding that OASIS could not rescue $\operatorname{Sec} 23 a$ expression in Bbf2 $\mathrm{h} 7^{-1-}$ chondrocytes. ${ }^{27}$

However, messenger RNA levels of another COPII component, SEC24D (MIM 607186), were significantly upregulated in the presence of functional OASIS protein $(P=0.007$ versus empty vector; $P=0.009$ versus VT312), but not when the Lys312del protein variant was present (Figure $4 \mathbf{b}$ ). Immunoblotting of whole-cell lysates from transfected cells was consistent with the expression studies (Figure 4c). More SEC24D protein was seen in cells overexpressing WT OASIS than in those expressing the nonfunctional VT312 OASIS or in the empty vector control (Figure $4 \mathbf{c}$,d), while SEC23A protein levels were unchanged with OASIS overexpression (Figure 4c,e). Without sufficient SEC24D expression, we suspect COPII formation is disrupted and osteoblasts cannot efficiently secrete type I collagen and other bone matrix proteins. While we did not see abnormal retention of type I procollagens or pro $\alpha$ chains in proband (VI-7) fibroblasts (Figure 1d), we would not expect to see evidence of defective secretion in the collagen analysis we performed, as CREB3L1 is not expressed in skin.

\section{OASIS target specificity}

The binding ability of many basic leucine zipper transcription factors is determined by the formation of homo- and/or heterodimers. It has been proposed that switching out the binding partners in these dimers could alter specificity. ${ }^{29}$ This 

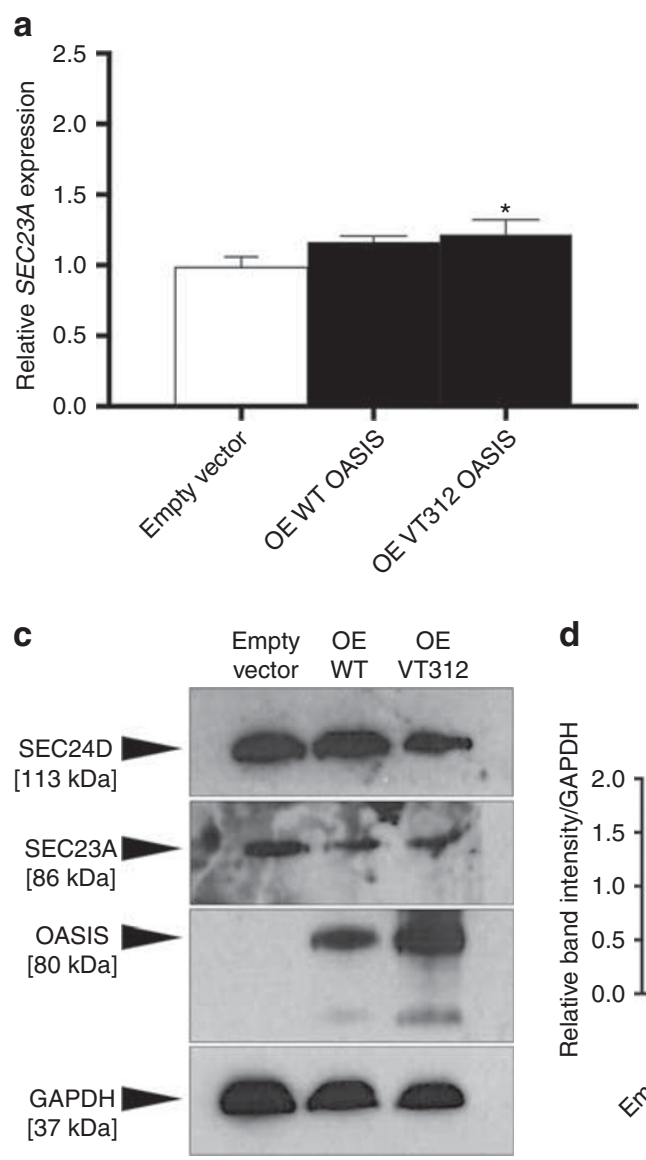

d b
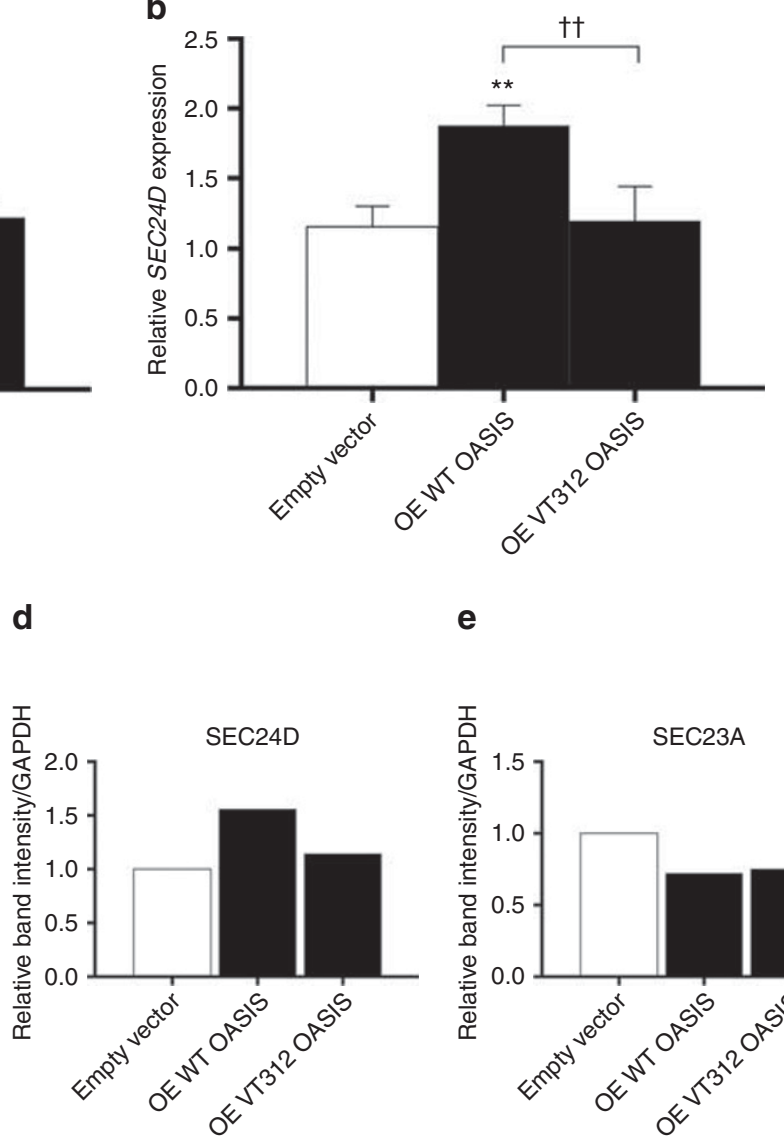

e

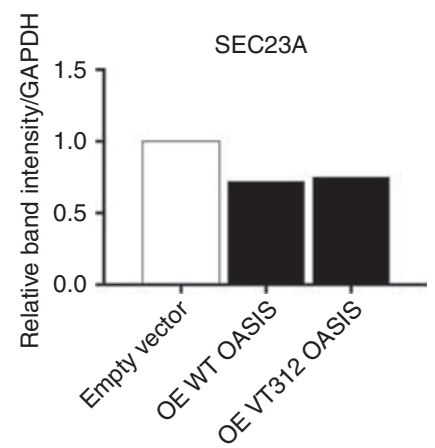

Figure 4 OASIS regulates expression of SEC24D, but not SEC23A. (a) SEC23A, encoding a component of the coat protein II complex, is not significantly upregulated in the presence of WT OASIS under normal conditions. Apparently increased expression in the presence of VT312 OASIS is significant $(P=0.03)$, but given that there is no significant difference in SEC23A expression between WT- and VT312-overexpressing cells, we conclude that SEC23A is not regulated by OASIS. ${ }^{*} P<0.05$ versus empty vector-transfected control (Tukey test). Quantitative reverse transcription polymerase chain reaction. (b) A gene encoding another component of the coat protein II complex, SEC24D, is significantly upregulated in the presence of WT OASIS $(P=0.007$ versus empty vector; $P=0.009$ versus VT312-transfected), but not in the presence of nonfunctional VT312 OASIS. $* \star P<0.01$ versus empty vector-transfected control. $+\uparrow P<0.01$ versus the indicated group (Tukey test). $Y$-axes represent relative messenger RNA expression normalized by the level of GAPDH. Values shown are the mean of three independent experiments $(n=3)$. Error bars, SD. Quantitative reverse transcription polymerase chain reaction. (c) Immunoblotting of whole-cell lysates from HEK293 overexpressing either WT or VT312 OASIS is consistent with the expression studies, showing that (d) the amount of SEC24D protein was augmented upon WT OASIS overexpression, while (e) SEC23A protein levels were static. OE, overexpressed; WT, wild type.

could help explain the variability of OASIS target genes in cell types in which expression has been reported. To examine whether OASIS forms homodimers to promote expression of SEC24D, we overexpressed WT and VT312 OASIS simultaneously at varying ratios (WT:VT312 ratios of $3: 1,1: 1,1: 3$ ) with the expectation that, if OASIS formed homodimers, the increasing presence of variant, nonfunctional OASIS protein would result in the abrogation of target gene expression. The presence of any WT OASIS altered SEC24D expression significantly over both empty vector control and VT312 overexpression alone, but there were no significant differences in SEC24D levels among cells transfected with the vectors at different ratios (Supplementary Figure S3). This suggested that if OASIS forms dimers, it partners with other proteins and not itself.

\section{DISCUSSION}

This is the second report of a family with OI-like phenotypes caused by mutation of CREB3L1 and confirms CREB3L1 as an OI-disease gene. Clues to the mechanisms by which alteration of OASIS function might result in OI come from looking at a closely related gene, CREB3L2. Knockout of Creb3l2 in mice resulted in severe chondrodysplasia due to failure to secrete type II procollagen with the resultant disruption of hypertrophic zone formation in cartilage tissue. Type II collagen and other cartilage matrix proteins accumulated in the ER lumen of Creb3l2 $2^{-1-}$ chondrocytes. ${ }^{27}$ The protein product of Creb3l2, Bbf2h7, promotes transcription of Sec23a, which encodes a component of the COPII complex. ${ }^{27}$ In human dermal fibroblasts, CREB3L2 knockdown suppressed SEC23A expression and caused Golgi dysmorphology (irregularly 
curved cisternae and aggregation), indicative of problems with subcellular trafficking. ${ }^{30}$ Molecular and cellular analysis of Creb3l1 knockout mice revealed similar cellular and tissue phenotypes in bone tissue, rather than in cartilage. Creb3l1 ${ }^{-\backslash-}$ primary osteoblasts had distended ERs full of bone matrix proteins (type I procollagen and osteocalcin) and there was significantly reduced collagen content in the bone matrix, leading to severe osteopenia and fractures. ${ }^{16}$ Because BBF2H7 is important for COPII formation and for secretion of type II collagen and other cartilage matrix proteins from chondrocytes during chondrogenesis, we proposed that OASIS has an analogous role in the secretion of type I collagen and other bone matrix proteins from osteoblasts during osteogenesis.

COPII complexes are involved in the trafficking of secreted proteins from the ER to the Golgi and are specifically involved in ER export. ${ }^{28}$ Carrier structure consists of an inner coat formed by SEC23 and SEC24 proteins and an outer coat consisting of SEC13 and SEC31 proteins, ${ }^{31}$ which are thought to confer stability. ${ }^{32}$ Vesicle formation is aided by SAR1, SEC12, and SEC16. ${ }^{31}$ Shuttling of large proteins, such as procollagen, requires the creation of extra-large COPII carriers. ${ }^{31}$ Auxiliary proteins TANGO1 (transport and Golgi organization 1) and cTAGE5 (cutaneous T-cell lymphomaassociated 5) are found at ER exit sites and help with loading of large COPII. ${ }^{31}$ They bind SEC23 and SEC24 proteins in the inner coat on the cytoplasmic side of the ER membrane and procollagen on the luminal side, both guiding procollagen into the forming vesicle and inhibiting association of the outer coat with the inner coat. This latter action delays vesicle fission, giving sufficient time for a larger vesicle to form. ${ }^{31}$ Sedlin, another helper protein, is recruited by TANGO1 and is thought to stabilize the inner coat and prevent premature membrane constrictions. ${ }^{31}$ The absence of COPII structural or helper proteins could result in hypoplastic carriers and the cell may be unable to secrete large proteins efficiently. We have shown that OASIS regulates the expression of the COPII component SEC24D.

The importance of secretory pathways in osteogenesis is already appreciated. Recessive mutations in SEC24D itself cause a syndromic form of OI with features that resemble those of Cole-Carpenter syndrome (MIM 616294): skull ossification defects, fractures. ${ }^{33}$ It is possible that OASIS lies upstream of additional components of the COPII complex and plays a larger role in construction of these carriers. We hypothesize that OASIS-mediated secretory pathways, specifically the OASIS-SEC24D pathway, are necessary for normal bone development. Furthermore, a recent report identified an $\mathrm{X}$-linked form of OI associated with mutations in membranebound transcription factor peptidase, site 2 (MBTPS2; MIM 300294), which encodes site- 2 metalloprotease. ${ }^{6}$ Site- 2 metalloprotease is a serine protease that cleaves OASIS in response to the sensing of ER stress, and thereby regulates OASIS activity. ${ }^{34,35}$ Taken together, the discovery of these novel disease genes demonstrates the critical contributions of secretory pathways to bone development and bone homeostasis (Supplementary Figure S4) and should give direction to continued efforts to identify the genetic causes of OI and other bone dysplasias.

Notably, expression of OASIS has also been reported in astrocytes, ${ }^{26}$ pancreatic $\beta$-cells, ${ }^{17}$ and goblet cells. ${ }^{18}$ Along with physiological ER stress pathways that involve OASIS (as others have hypothesized), ${ }^{16,29}$ secretory pathways involving OASIS may be unique to "professional" secretory cell types in which the demand for protein production is especially high ${ }^{36}$ and may be cytoprotective. Given the suspected importance of OASIS for these tissues, it is notable that none of the individuals in the family appear to have neural, pancreatic, or intestinal abnormalities. Heterozygosity for the deletion may be insufficient to cause major abnormalities in these tissues, unlike in bone. It is possible evidence of such abnormalities would be found in homozygous individuals if they survived longer. Future studies will further elucidate the importance of OASIS for the development of bone and other tissue types.

\section{SUPPLEMENTARY MATERIAL}

Supplementary material is linked to the online version of the paper at http://www.nature.com/gim

\section{ACKNOWLEDGMENTS}

This work was supported by the Freudmann Research Fund at the University of Washington, the National Institute of Arthritis and Musculoskeletal and Skin Diseases (F31AR069971), a Cell \& Molecular Biology Training Grant (5T32GM007270), the Molecular Medicine Training Grant (5T32GM095421), the University of Washington Department of Pathology, and the Collagen Diagnostic Laboratory. Sequencing was provided by the University of Washington Center for Mendelian Genomics funded by the National Human Genome Research Institute and the National Heart, Lung, and Blood Institute (2UM1HG006493). We sincerely thank the family for their research consent. Thanks to Diana Chen (University of Washington) for performing the collagen analysis and William Mahoney (University of Washington) for his biochemical wisdom in troubleshooting the EMSA.

\section{DISCLOSURE}

The authors declare no conflict of interest.

\section{REFERENCES}

1. Rauch F, Glorieux FH. Osteogenesis imperfecta. Lancet 2004;363: 1377-85.

2. Byers $P$, Pyott $S$. Recessively inherited forms of osteogenesis imperfecta. Annu Rev Genet 2012;46:475-97.

3. Harrington J, Sochett E, Howard A. Update on the evaluation and treatment of osteogenesis imperfecta. Pediatr Clin N Am 2014;61: 1243-57.

4. Semler O, Garbes L, Keupp K, et al. A mutation in the 5'-UTR of IFITM5 creates an in-frame start codon and causes autosomal-dominant osteogenesis imperfecta type $\mathrm{V}$ with hyperplastic callus. Am J Hum Genet 2012;91:349-57.

5. Guillen-Navarro E, Ballesta-Martinez MJ, Valencia M, et al. Two mutations in IFITM5 causing distinct forms of osteogenesis imperfecta. Am J Med Genet A 2014;164A:1136-42. 
6. Lindert U, Cabral WA, Ausavarat $S$, et al. MBTPS2 mutations cause defective regulated intramembrane proteolysis in $\mathrm{X}$-linked osteogenesis imperfecta. Nat Commun 2016;7:11920.

7. Symoens S, Malfait F, D'hondt S, et al. Deficiency for the ER-stress transducer OASIS causes severe recessive osteogenesis imperfecta in humans. Orphanet J Rare Dis 2013;8:e154.

8. Pyott SM, Tran TT, Leistritz DF, et al. WNT1 mutations in families affected by moderately severe and progressive recessive osteogenesis imperfecta. Am J Hum Genet 2013;92:590-7.

9. Bonadio J, Holbrook KA, Gelinas RE, Jacob J, Byers PH. Altered triple helical structure of type I procollagen in lethal perinatal osteogenesis imperfecta. J Biol Chem 1985;260:1734-42.

10. ThermoScientific. Tech tip \#72: PCR protocol for generating optimized templates for pierce human in vitro expression kits. 2017. https://tools. thermofisher.com/content/sfs/brochures/TR0072-IVT-PCR-protocol.pdf. Accessed 28 February 2017.

11. Livak KJ, Schmittgen TD. Analysis of relative gene expression data using real-time quantitative $P C R$ and the $2 \wedge($-delta delta $C(T)$ ) method. Methods 2001;25:402-8.

12. Rasdan WS. ImageJ. Release $1.51 \mathrm{j}$. US National Institutes of Health: Bethesda, MD, 2017.

13. Davydov EV, Goode DL, Sirota M, Cooper GM, Sidow A, Batzoglou S. Identifying a high fraction of the human genome to be under selective constraint using GERP++. PLoS Comput Biol 2010;6:e1001025.

14. Siepel A, Bejerano G, Pederson JS, et al. Evolutionarily conserved elements in vertebrate, insect, worm, and yeast genomes. Genome Res 2005; 15:1034-50.

15. Kondo S, Murakami T, Tatsumi K, et al. OASIS, a CREB/ATF-family member, modulates UPR signalling in astrocytes. Nat Cell Biol 2005;7:186-94.

16. Murakami $T$, Saito $A$, Hino $S$, et al. Signalling mediated by the endoplasmic reticulum stress transducer OASIS is involved in bone formation. Nat Cell Biol 2009;11:1205-11.

17. Vellanki RN, Zhang L, Guney MA, Rocheleau JV, Gannon M, Volchuk A. OASIS/CREB3L1 induces expression of genes involved in extracellular matrix production but not classical endoplasmic reticulum stress response genes in pancreatic $\beta$-cells. Endocrinology 2010;151:4146-57.

18. Asada R, Saito A, Kawasaki N, et al. The endoplasmic reticulum stress transducer OASIS is involved in the terminal differentiation of goblet cells in the large intestine. J Biol Chem 2012;287:8144-53.

19. Yang Q, Yin R, Cao X, Wu D, Chen W, Zhou Y. Association of two polymorphisms in the FADS1/FADS2 gene cluster and the risk of coronary artery disease and ischemic stroke. Int J Clin Exp Pathol 2015;8:7318-31.

20. Wanschers BFJ, Szklarczyk R, van den Brand MAM, et al. A mutation in the human CBP4 ortholog UQCC3 impairs complex III assembly, activity and cytochrome b stability. Hum Mol Genet 2014;23:6356-65.

21. Smith KR, Dahl HM, Canafoglia L, et al. Cathepsin F mutations cause type B Kufs disease, an adult-onset neuronal ceroid lipofuscinosis. Hum Mol Genet 2013;22:1417-23.
22. Martin-Alonso M, Garcia-Redondo AB, Guo D, et al. Deficiency of MMP17/MT4-MMP proteolytic activity predisposes to aortic aneurysm in mice. Circ Res 2015;117:e13-26.

23. Sohail A, Sun Q, Zhao H, Bernardo MM, Cho J, Fridman R. MT4-(MMP17) and MT6-MMP (MMP25), a unique set of membrane-anchored matrix metalloproteinases: properties and expression in cancer. Cancer Metastasis Rev 2008;27:289-302.

24. Wang $Y, Y u$ S, Li Y, Luo H. Expression and clinical significance of matrix metalloproteinase-17 and -25 in gastric cancer. Oncol Lett 2015;9: 671-76.

25. Kosugi S, Hasebe M, Tomita M, Yanagawa H. Systematic identification of cell cycle-dependent yeast nucleocytoplasmic shuttling proteins by prediction of composite motifs. Proc Natl Acad Sci U S A 2009;106: 10171-6.

26. Honma $Y$, Kanazawa K, Mori T, et al. Identification of a novel gene, OASIS, which encodes for a putative CREB/ATF family transcription factor in the long-term cultured astrocytes and gliotic tissue. Mol Brain Res 1999;69:93-103.

27. Saito A, Hino S, Murakami T, et al. Regulation of endoplasmic reticulum stress response by a BBF2H7-mediated Sec23a pathway is essential for chondrogenesis. Nat Cell Biol 2009;11:1197-204.

28. Canty EG, Kadler KE. Procollagen trafficking, processing and fibrillogenesis. J Cell Sci 2005;118:1341-53.

29. Asada R, Kanemoto $S$, Kondo S, Saito A, Imaizumi $K$. The signalling from endoplasmic reticulum-resident bZIP transcription factors involved in diverse cellular physiology. J Biochem 2011;149:507-18.

30. Ishikura-Kinoshita S, Saeki H, Tsuji-Naito K. BBF2H7-mediated Sec23A pathway is required for endoplasmic reticulum-to-Golgi trafficking in dermal fibroblasts to promote collagen synthesis. J Invest Dermatol 2012;132:2010-8.

31. Unlu G, Levic DS, Melville DB, Knapik EW. Trafficking mechanisms of extracellular matrix macromolecules: insights from vertebrate development and human diseases. Int J Biochem Cell Biol 2014;47: 57-67.

32. Copic A, Latham CF, Horlbeck MA, D'Arcangelo JG, Miller EA. ER cargo properties specify a requirement for COPII coat rigidity mediated by Sec13p. Science 2012;335:1359-62.

33. Garbes L, Kim K, Rieß A, et al. Mutations in SEC24D, encoding a component of the COPII machinery, cause a syndromic form of osteogenesis imperfecta. Am J Hum Genet 2015;96:432-9.

34. Omori Y, Imai J, Suzuki Y, Watanabe S, Tanigami A, Sugano S. OASIS is a transcriptional activator of CREB/ATF family with a transmembrane domain. Biochem Biophys Res Commun 2002;293:470-77.

35. Murakami T, Kondo S, Ogata M, et al. Cleavage of the membrane-bound transcription factor OASIS in response to endoplasmic reticulum stress. J Neurochem 2006;96:1090-100.

36. Wu J, Kaufman RJ. From acute ER stress to physiological roles of the unfolded protein response. Cell Death Differ 2006;13:374-84. 